\title{
ABC Transporter
}

National Cancer Institute

\section{Source}

National Cancer Institute. ABC Transporter. NCI Thesaurus. Code C17750.

A family of transmembrane proteins that usually function as dimers to hydrolyze ATP, which permits them to transport a wide variety of substrates across cellular membranes. These proteins consist of two distinct domains, a transmembrane domain and a nucleotide-binding domain. ATP binding is required for both dimerization and transporter activity. 